\title{
Sudden Future Singularities and their observational signatures in Modified Gravity
}

\section{Andreas Lymperis*}

Department of Physics, University of Patras, 26500 Patras, Greece

E-mail: alymperis@upatras.gr

\begin{abstract}
We verify the existence of Generalized Sudden Future Singularities (GSFS) in quintessence models with scalar field potential of the form $V(\phi) \sim|\phi|^{n}$ where $0<n<1$ and in the presence of a perfect fluid, both numerically and analytically, using a proper generalized expansion ansatz for the scale factor and the scalar field close to the singularity. This generalized ansatz includes linear and quadratic terms, which dominate close to the singularity and cannot be ignored when estimating the Hubble parameter and the scalar field energy density; as a result, they are important for analysing the observational signatures of such singularities. We derive analytical expressions for the power (strength) of the singularity in terms of the power $n$ of the scalar field potential. We then extend the analysis to the case of scalar tensor quintessence models with the same scalar field potential in the presence of a perfect fluid, and show that a Sudden Future Singularity (SFS) occurs in this case. We derive both analytically and numerically the strength of the singularity in terms of the power $n$ of the scalar field potential.
\end{abstract}

Corfu Summer Institute 2017 'School and Workshops on Elementary Particle Physics and Gravity' 2-28 September 2017

Corfu, Greece

\footnotetext{
*Speaker.
} 


\section{Introduction}

Latest evidence of an accelerating Universe [1, 2, 3, 4, 5, 6], has opened new windows in the context of the study of physics in cosmological scales, and has lead to the consideration of models alternative to $\Lambda \mathrm{CDM}$. Such models include modifications of GR (modified Gravity) [7, 8], scalar field dark energy (quintessence) $[9,10]$, physically motivated forms of fluids $e . g$. Chaplygin gas $[11,12,38]$ etc.

Some of these dark energy models predict the existence of exotic cosmological singularities, involving divergences of the scalar spacetime curvature and/or its derivatives. These singularities can be either geodesically complete [13, 14, 15, 16, 34, 35, 36, 37, 39] (geodesics continue beyond the singularity and the Universe may remain in existence) or geodesically incomplete $[17,18,33]$ (geodesics do not continue beyond the singularity and the Universe ends at the classical level). They appear in various physical theories such as superstrings [19], scalar field quintessence with negative potentials [20], modified gravities and others [21, 22].

The divergence of the scale factor and/or its derivatives leads to divergence of scalar quantities like the Ricci scalar, thus to different types of singularities or 'cosmological milestones' [23, 25, 26]. However geodesics do not necessarily end at these singularities and if the scale factor remains finite, they are extended beyond these events [22] even though a diverging impulse may lead to dissociation of all bound systems in the Universe at the time $t_{s}$ of these events[24].

Thus, singularities can be classified [27] according to the behaviour of the scale factor $a(t)$, and/or its derivatives at the time $t_{s}$ of the event or equivalently, and the energy density and pressure of the content of the universe at the time $t_{s}$. A classification of such singularities and their properties is shown in Table 1.

Table 1: Classification and properties of cosmological singularities.

\begin{tabular}{cccccccccc}
\hline Name & $t_{\text {sing }}$ & $a\left(t_{s}\right)$ & $\rho\left(t_{s}\right)$ & $p\left(t_{s}\right)$ & $\dot{p}\left(t_{s}\right)$ & $w\left(t_{s}\right)$ & $\mathrm{T}$ & $\mathrm{K}$ & Geodesically \\
\hline \hline Big-Bang (BB) & 0 & 0 & $\infty$ & $\infty$ & $\infty$ & finite & strong & strong & incomplete \\
\hline Big-Rip (BR) & $t_{s}$ & $\infty$ & $\infty$ & $\infty$ & $\infty$ & finite & strong & strong & incomplete \\
\hline Big-Crunch (BC) & $t_{s}$ & 0 & $\infty$ & $\infty$ & $\infty$ & finite & strong & strong & incomplete \\
\hline Little-Rip (LR) & $\infty$ & $\infty$ & $\infty$ & $\infty$ & $\infty$ & finite & strong & strong & incomplete \\
\hline Pseudo-Rip (PR) & $\infty$ & $\infty$ & finite & finite & finite & finite & weak & weak & incomplete \\
\hline Sudden Future (SFS) & $t_{s}$ & $a_{s}$ & $\rho_{s}$ & $\infty$ & $\infty$ & finite & weak & weak & complete \\
\hline Big-Brake (BBS) & $t_{s}$ & $a_{s}$ & 0 & $\infty$ & $\infty$ & finite & weak & weak & complete \\
\hline Finite Sudden Future (FSF) & $t_{s}$ & $a_{s}$ & $\infty$ & $\infty$ & $\infty$ & finite & weak & strong & complete \\
\hline Generalized Sudden Future (GSFS) & $t_{s}$ & $a_{s}$ & $\rho_{s}$ & $p_{s}$ & $\infty$ & finite & weak & strong & complete \\
\hline Big-Separation (BS) & $t_{s}$ & $a_{s}$ & 0 & 0 & $\infty$ & $\infty$ & weak & weak & complete \\
\hline w-singularity (w) & $t_{s}$ & $a_{s}$ & 0 & 0 & 0 & $\infty$ & weak & weak & complete \\
\hline
\end{tabular}

A particularly interesting type of singularities are the Sudden Future Singularities [21], which involve violation of the dominant energy condition $\rho \geq|p|$, and divergence of the cosmic pressure of the Ricci Scalar and of the second time derivative of the cosmic scale factor Table 1. The scale factor can be parametrized as

$$
a(t)=\left(\frac{t}{t_{s}}\right)^{m}\left(a_{s}-1\right)+1-\left(1-\frac{t}{t_{s}}\right)^{q},
$$


where $a_{s}$ is the scale factor at the time $t_{s}$ and $1<q<2$. For this range of the parameter $q$, the scale factor and its first derivative, i.e. $a, \dot{a}$ respectively, and $\rho$ remain finite at $t_{s}$. However, the quantities $p, \dot{\rho}$ and $\ddot{a}$ become infinite. Thus, when the first derivative of the scale factor is finite at the singularity, but the second derivative diverges (SFS singularity [21, 28]), the energy density is finite but the pressure diverges.

In the following, we focus on the quintessence models with a perfect fluid, and investigate the strength of the GSFS both analytically and numerically. We extend the analysis to the case of scalar-tensor quintessence and investigate the modification of the strength of the singularity both analytically (using a proper expansion ansatz) and numerically, by explicitly solving the dynamical cosmological equations.

\section{The setup}

In FRW spacetime with metric

$$
d s^{2}=-d t^{2}+a^{2}(t)\left[\frac{d r^{2}}{1-k r^{2}}+r^{2}\left(d \theta^{2}+\sin ^{2} \theta d \phi^{2}\right)\right]
$$

the most general action involving gravity, nonminimally coupled with a scalar field $\phi$, and a perfect fluid is

$$
S=\int\left[\frac{1}{2} F(\phi) R+\frac{1}{2} g^{\mu v} \phi_{; \mu} \phi_{; v}-V(\phi)+\mathscr{L}_{(\text {fluid })}\right] \sqrt{-g} d^{4} x .
$$

where $F(\phi)$ is the nonminimal coupling of gravity to the scalar field and $\mathscr{L}_{\text {(fluid) }}$ the fluid term. We have set $8 \pi G=c=1$ and assume spatial flatness $(k=0)$. In the case of the scalar-tensor models, corresponding to the action (2.2), we assume a non-minimal coupling linear in the scalar field $F(\phi)=1-\lambda \phi$, even though the results on the type of the singularity in this class of models are unaffected by the particular choice of the non-minimal coupling.

In the special case where the non-minimal coupling $F(\phi)=1$, the action (2.2) reduces to the simple case of quintessece models with a perfect fluid

$$
S=\int\left[\frac{1}{2} R+\frac{1}{2} g^{\mu v} \phi_{; \mu} \phi_{; v}-V(\phi)+\mathscr{L}_{(\text {fluid })}\right] \sqrt{-g} d^{4} x
$$

The potential $V(\phi)$ is of the form

$$
V(\phi)=A|\phi|^{n}, \quad A>0,
$$

with $0<n<1$ and $A$ a constant parameter. The dynamical evolution of the scalar field due to the potential is shown in Fig. 1

It was shown, through a qualitative analysis [30], that the power law scalar potential (2.4) leads to singularities at any scale factor derivative order larger than three, depending on the value of the power $n$. In particular, for $k<n<k+1$, with $k>0$, the $(k+2)^{t h}$ derivative of the scale factor diverges at the singularity. This is in fact the simplest extension of $\Lambda$ CDM with geodesically complete cosmic singularities and occurs at the time $t_{s}$, when the scalar field becomes zero $(\phi=0)$. 


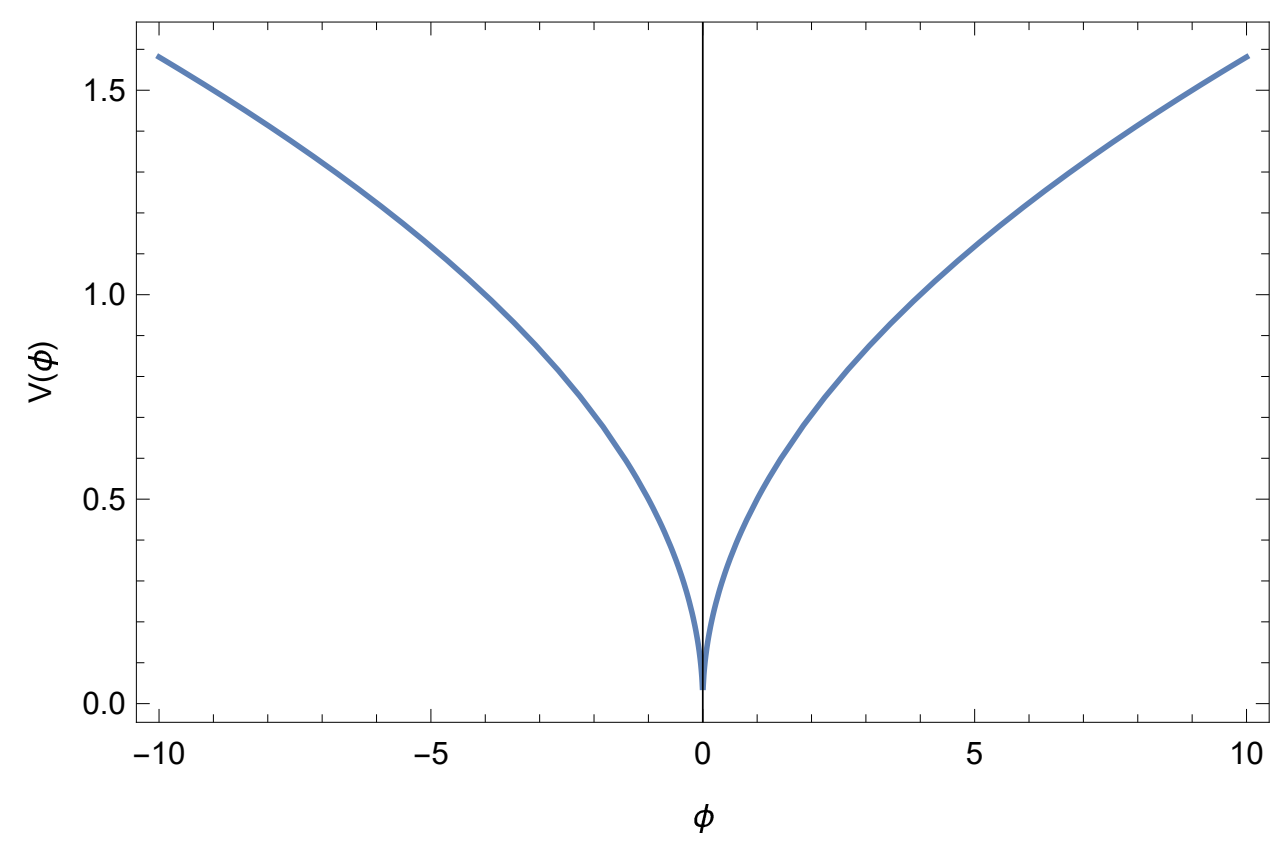

Figure 1: Dynamical evolution of the scalar field potential $V(\phi)=A|\phi|^{n}$

\section{The Quintessence case}

The action in this class of models, is of the form (2.3). The energy density and pressure of the scalar field $\phi$, may be written as

$$
\rho_{\phi}=\frac{1}{2} \dot{\phi}^{2}+V(\phi) \quad \text { and } \quad p_{\phi}=\frac{1}{2} \dot{\phi}^{2}-V(\phi) .
$$

and we assume that the perfect fluid is pressureless $\left(p_{m}=0\right)$.

Variation of the action (2.3) leads to the dynamical equations

$$
\begin{gathered}
3 H^{2}=\frac{3 \Omega_{0, m}}{a^{3}}+\frac{1}{2} \dot{\phi}^{2}+V(\phi) \\
\ddot{\phi}=-3 H \dot{\phi}-A n|\phi|^{n-1} \Theta(\phi) \\
2 \dot{H}=-\frac{3 \Omega_{0, m}}{a^{3}}-\dot{\phi}^{2}
\end{gathered}
$$

where $a$ is the scale factor, $H=\frac{\dot{a}}{a}$ is the Hubble parameter, $\rho_{m}=\frac{\rho_{0, m}}{a^{3}}=\frac{3 \Omega_{0, m}}{a^{3}}, \Omega_{0, m}=0.3$ and

$$
\Theta(\phi)= \begin{cases}1, & \phi>0 \\ -1, & \phi<0\end{cases}
$$

From eqs (3.2), (3.4), it follows that when $t \rightarrow t_{s}$ i.e. $\phi \rightarrow 0$, the Hubble parameter $H$ and its first derivative $\dot{H}$ remain finite and so does $\dot{\phi}$. But in eq. (3.3) there is a divergence of the term 
$\phi^{n-1}$ for $0<n<1$ and thus $\ddot{\phi} \rightarrow \infty$ as $\phi \rightarrow 0$. $\ddot{H}$ also diverges at this point due to the divergence of $\ddot{\phi}$, as follows by differentiating eq. (3.4). This implies that the third derivative of the scale factor diverges, and a GSFS occurs at this point (i.e. $a_{s}, \rho_{s}, p_{s}$ remain finite but $\dot{p} \rightarrow \infty$ ). Thus, the constraints on the power exponents $q, r$ of the diverging terms in the expansion of the scale factor $\left(\sim\left(t_{s}-t\right)^{q}\right)$ and of the scalar field $\left(\sim\left(t_{s}-t\right)^{r}\right)$ are $2<q<3$ and $1<r<2$ respectively (see eqs (3.8), (3.9) below). It has been shown in [31] that by choosing $q$ to lie in the intervals $(N, N+1)$ for $N \geq 2$, where $N \in \mathbb{Z}^{+}$, a finite-time singularity occurs in which

$$
\frac{d^{N+1} a}{d t^{N+1}} \rightarrow \infty
$$

but

$$
\frac{d^{s} a}{d t^{s}} \rightarrow 0, \text { for } s \leq N \in \mathbb{Z}^{+}
$$

This allows for pressure singularities which are accompanied by divergence of higher time derivatives of the scale factor (divergence of the fourth-order derivative of the scale factor [31] when $p \rightarrow \infty$ ), in Friedmann solutions of higher-order gravity $(f(R))$ theories [32].

The above qualitative analysis can be extended to a quantitative level by introducing a new ansatz for the scale factor and the scalar field, containing linear and quadratic terms of $\left(t_{s}-t\right)$. These terms play an important role, since they dominate in the first and second derivative of the scale factor as the singularity is approached.

The new ansatz for the scale factor which generalizes (1.1), by introducing linear and quadratic terms in $\left(t_{s}-t\right)$, is of the form [29]

$$
a(t)=1+\left(a_{s}-1\right)\left(\frac{t}{t_{s}}\right)^{m}+b\left(t_{s}-t\right)+c\left(t_{s}-t\right)^{2}+d\left(t_{s}-t\right)^{q}
$$

where $m=\frac{2}{3(1+w)}, w$ the state parameter, $b, c, d$ are real constants to be determined, and $2<q<3$ so that $\dddot{a}$ diverges at the GSFS.

The corresponding expansion of the scalar field $\phi(t)$ in the vicinity of the singularity is of the form

$$
\phi(t)=f\left(t_{s}-t\right)+h\left(t_{s}-t\right)^{r}
$$

where $1<r<2$ so that $\ddot{\phi}$ diverges at the singularity and $f, h$ are real constants to be determined.

From eq. (3.3) and differentiated eq. (3.4), using the forms of the scale factor (3.8) and the scalar field (3.9), we get two equations that contain only dominant terms in $\left(t_{s}-t\right)$, in which both the left and right-hand sides diverge at the singularity for $0<n<1,2<q<3$ and $1<r<2$. Equating the power laws $q$ and $r$ of the divergent terms we obtain

$$
\begin{aligned}
& r=n+1 \\
& q=r+1 .
\end{aligned}
$$

and it follows that

$$
q=n+2
$$




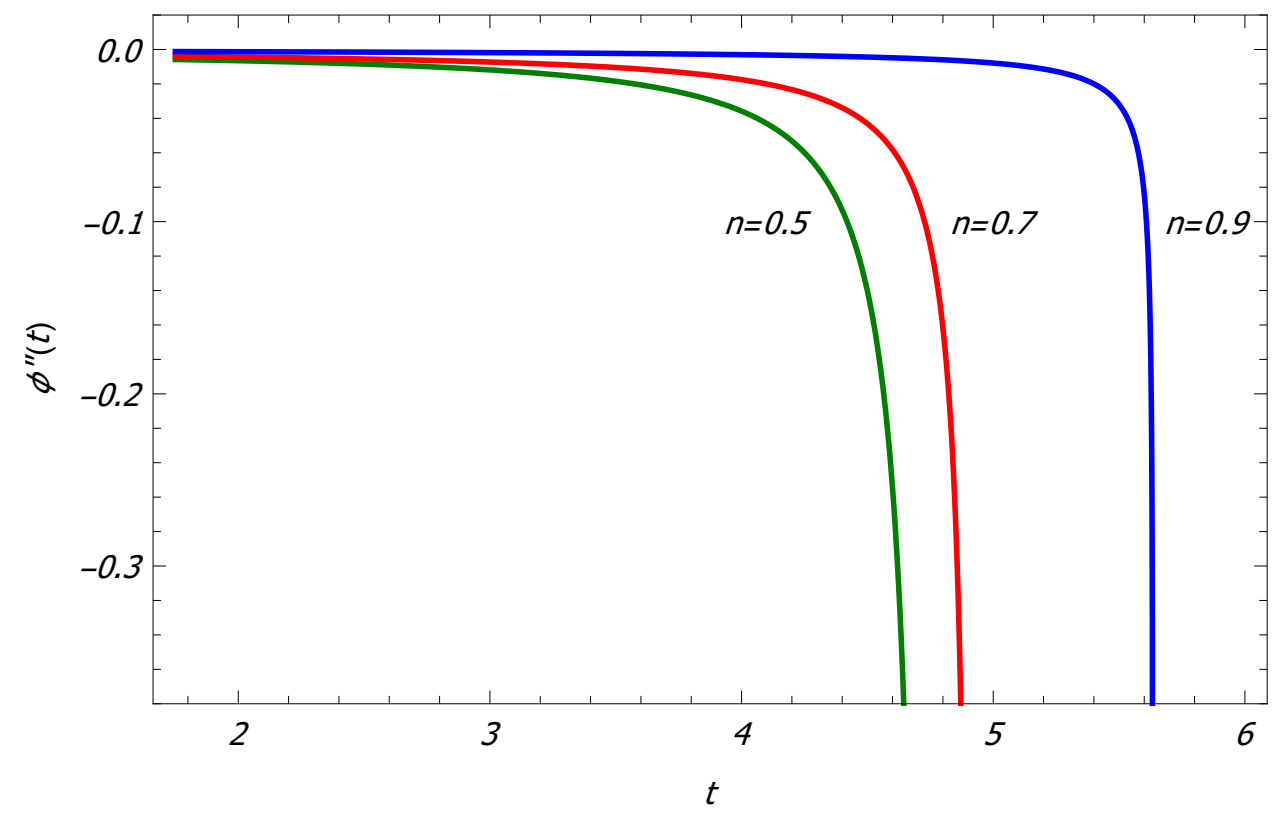

Figure 2: Numerical solutions of the second time derivative of the scalar field for $n=0.5,0.7,0.9$. Notice the divergence at the time of the singularity when the scalar field vanishes.

Figure 2 shows the divergence of the second derivative of the scalar field at the time of the singularity. In figures $3 \mathrm{a}, 3 \mathrm{~b}$ we plot the numerically verified derived power law dependence (eqs (3.10), (3.12)) of the scalar field and the scale factor respectively, as the singularity is approached. It is clear that eqs (3.10), (3.12) are consistent with the qualitatively expected range of $r, q$, for $0<n<1$.

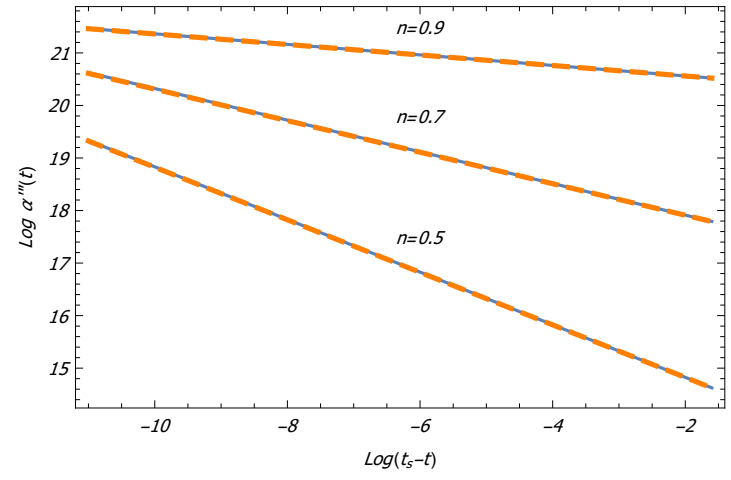

(a) $3 \mathrm{a}$

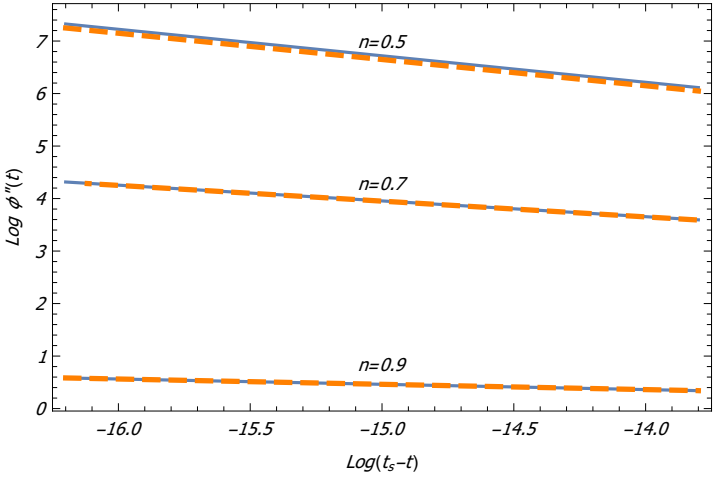

(b) $3 \mathrm{~b}$

Figure 3: Plots of numerical verification of the $q$-exponent (3a) and $r$-exponent (3b) for 3 values of $n(n=0.5, n=0.7$ and $n=0.9)$. The orange dashed line, denotes the analytical, while the blue line denotes the numerical solution. As expected the slopes for each $\mathrm{n}$ for both $q$ and $r$ are identical.

The additional linear and quadratic terms in $\left(t_{s}-t\right)$, in the expression of the scale factor (3.8), play an important role in the estimation of the Hubble parameter and its derivative as the singularity is aproached. An interesting result arises from the derivation of the relation between the coefficients 
$b, c$. The relations between these coefficients can lead to relations between the Hubble parameter and its derivative close to the singularity, which in turn correspond to observational predictions, that may be used to identify the presence of these singularities in angular diameter of luminosity distance data. The relation between $b, c$ is of the form

$$
c=\frac{\rho_{0, m}}{4 a_{s}^{2}}-\frac{1}{2}\left(a_{s}-1\right) m(m-1)-\frac{\left[\left(a_{s}-1\right) m-b\right]^{2}}{a_{s}},
$$

and thus

$$
\dot{H}=\frac{3 \Omega_{0, m}}{2 a_{s}^{3}}-3 H^{2}
$$

and as a function of redshift parameter $z$ at present time

$$
H^{2}(z)=\Omega_{0, m}(1+z)^{3}\left[1-(1+z)^{3}\left(1+z_{0}\right)^{-3}\right]+(1+z)^{6}\left(1+z_{0}\right)^{-6} H_{0}^{2},
$$

where $H_{0}, z_{0}$ are the Hubble and redshift parameter respectively at present time. This result may be used as observational signature of such singularities in this class of models.

In the absence of the perfect fluid, the strength of the singularity remains unaffected. This means that the evaluated relations of $r$ and $q$ (eqs (3.10), (3.12)) respectively, are exactly the same. The Hubble parameter and its derivative in this case is

$$
\dot{H}=-3 H^{2}
$$

and as a function of redshift parameter $z$ at present time

$$
H(z)=\frac{H_{0}(1+z)^{3}}{\left(1+z_{0}\right)^{3}}
$$

These are the reduced relations of eqs (3.14) and (3.15) respectively, for $\rho_{0, m}=0$.

\section{Modified Gravity: The Scalar-Tensor Quintessence case}

The action of the theory, in this class of models, is of the form (2.2). The corresponding dynamical equations are

$$
\begin{gathered}
3 F H^{2}=\frac{3 \Omega_{0, m}}{a^{3}}+\frac{\dot{\phi}^{2}}{2}+V-3 H \dot{F} \\
\ddot{\phi}+3 H \dot{\phi}-3 F_{\phi}\left(\frac{\ddot{a}}{a}+H^{2}\right)+A n|\phi|^{(n-1)} \Theta(\phi)=0 \\
-2 F\left(\frac{\ddot{a}}{a}-H^{2}\right)=\frac{3 \Omega_{0, m}}{a^{3}}+\dot{\phi}^{2}+\ddot{F}-H \dot{F},
\end{gathered}
$$

where $F_{\phi}=\frac{d F}{d \phi}$. From eq. (4.1), it is clear that $H, \dot{\phi}, F, \dot{F}$ all remain finite when $\phi \rightarrow 0\left(t \rightarrow t_{s}\right)$. However, in eq. (4.2) there is a divergence of the term $V_{\phi}$ for $0<n<1$ and $\ddot{\phi} \rightarrow \infty$ as $\phi \rightarrow 0$. This means that $\ddot{F} \rightarrow \infty$ because of the generation of the second derivative of $\phi$ that leads to a 
divergence of $\ddot{a}$ in eq. (4.3). Clearly, an SFS singularity (Table 1) is expected to occur in scalartensor quintessence models, as opposed to the GSFS singularity in the corresponding quintessence models. Thus, the constraints on the power exponents $q, r$ in this case are $1<q<2$ and $1<r<2$ respectively.

From the above dynamical equations, using the same parametrizations (3.8), (3.9) for the scale factor and the scalar field respectively and keeping only the dominant terms, the values for $r$ and $q$ are

$$
\begin{gathered}
q=r \\
r=n+1,
\end{gathered}
$$

which leads to

$$
q=n+1
$$

In figures $4 \mathrm{a}, 4 \mathrm{~b}$ we illustrate the numerically verified derived power law dependence eqs (4.5), (4.6) of the scalar field and the scale factor respectively, as the singularity is approached. Figures $5 \mathrm{a}, 5 \mathrm{~b}$ depict the divergence of the second derivative, of both the scale factor and the scalar field, at the time of the singularity.

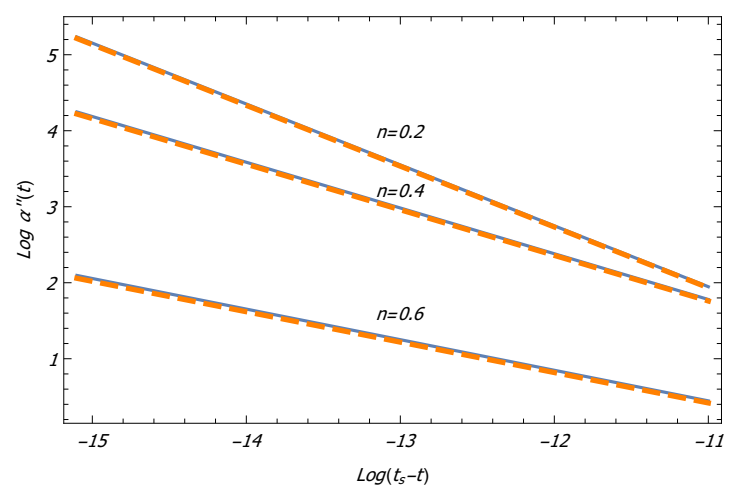

(a) $4 \mathrm{a}$

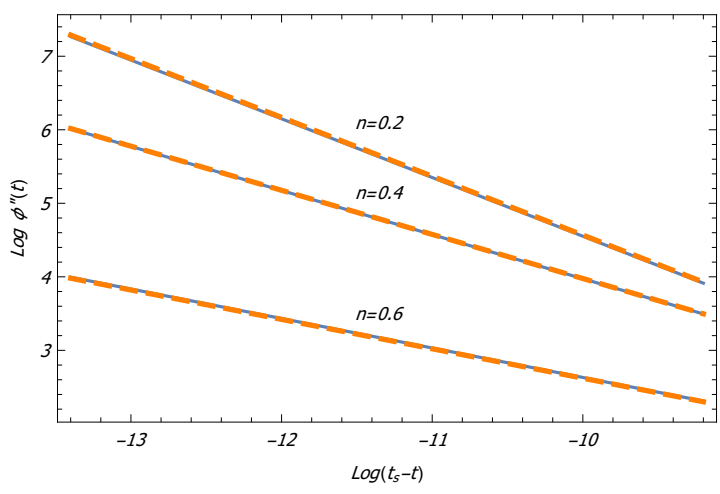

(b) $4 \mathrm{~b}$

Figure 4: Numerical verification of the $q$-exponent (4a) and $r$-exponent (4b), in the scalar-tensor case, for 3 values of $n(n=0.2, n=0.4$ and $n=0.6)$. The orange dashed line, denotes the analytical, while the blue line denotes the numerical solution. As expected the slopes for each $\mathrm{n}$ for both $q$ and $r$ are identical.

The results (4.5) and (4.6) are consistent with the above qualitative discussion for the expected strength of the singularity. Thus, in the case of the scalar-tensor theory, we have a stronger singularity at $t_{s}$, as compared to the singularity that occurs in quintessence models. This is a general result, valid not only for the coupling constant of the form $F=1-\lambda \phi$ but also for other forms of $F(\phi)$ (e.g. $F \sim \phi^{r}$ ), because the second derivative of $F$ with respect to time, in the dynamical equations, will always generate a second derivative of $\phi$ with divergence, leading to a divergence of $\ddot{a}$. 


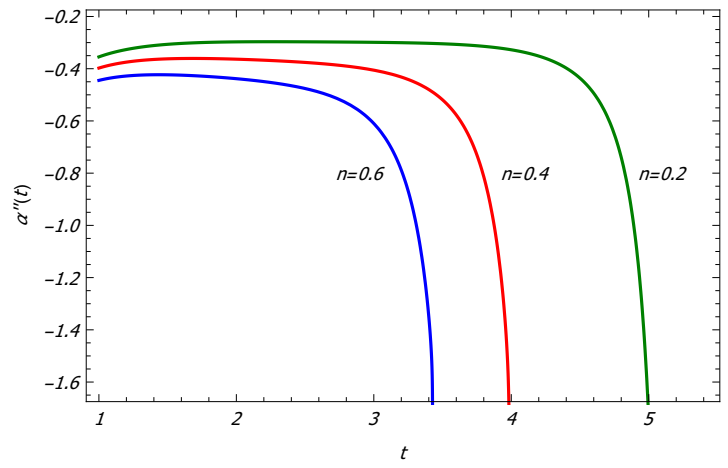

(a) $5 \mathrm{a}$

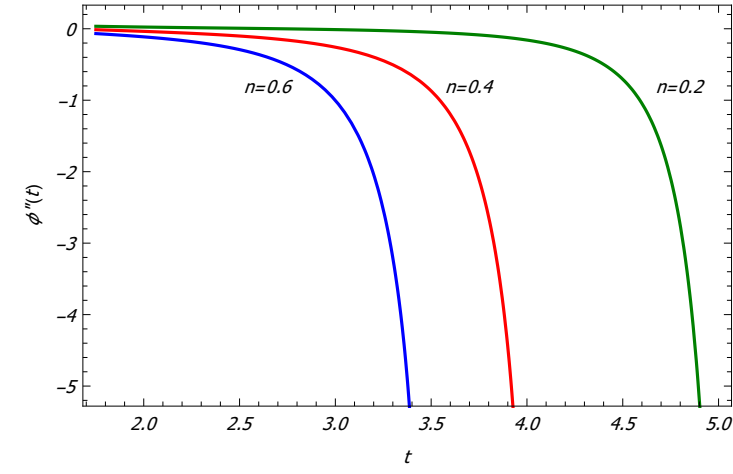

(b) $5 b$

Figure 5: Numerical solutions of the second time derivative of the scale factor (5a) and the scalar field ( $5 \mathrm{~b}$ ) for $n=0.2,0.4,0.6$. Notice the divergence of both the scale factor and scalar field at the time of the singularity.

The quadratic term of $\left(t_{s}-t\right)$, in the expression of the scale factor (3.8), is now subdominant as the second derivarive of the scale factor diverges. The only additional term of $\left(t_{s}-t\right)$ that can play an important role in the estimation of the Hubble parameter, is the linear term. Clearly, for the first derivative of (3.8), as $t \rightarrow t_{s}$ from below, the linear term dominates over all other terms, while the quadratic term is subdominant in the second derivative, in the divergence of the $q$-term. Thus, in the case of the scalar-tensor quintessence models $H$ remain finite and dominated by the term $b\left(t_{s}-t\right)$, while $\dot{H} \rightarrow \infty$ as $t \rightarrow t_{s}$.

As in quintessence case of the previous section, in the absence of the perfect fluid, the strength of the singularity remains unaffected. This means that the evaluated relations of $r$ and $q$, eqs (4.5), (4.6) respectively, are exactly the same.

\section{Conclusions and Discussion}

We have derived analytically and numerically the cosmological solution close to a future-time singularity for both quintessence and scalar-tensor quintessence models. For quintessence, we have shown that there is a divergence of $\dddot{a}$ and a GSFS singularity occurs $\left(a_{s}, \rho_{s}, p_{s}\right.$ remain finite but $\dot{p} \rightarrow \infty)$, while in the case of scalar-tensor quintessence models there is a divergence of $\ddot{a}$ and an SFS singularity occurs $\left(a_{s}, \rho_{s}\right.$ remain finite but $\left.p_{s} \rightarrow \infty, \dot{p} \rightarrow \infty\right)$. In the absence of the perfect fluid in the dynamical equations, in both cases, we have shown that this result is still valid in our cosmological solution.

These are the simplest non-exotic physical models where GSFS and SFS singularities naturally arise. In the case of scalar-tensor quintessence models, there is a divergence of the scalar curvature $R=6\left(\frac{\ddot{a}}{a}+\frac{\dot{a}^{2}}{a^{2}}\right) \rightarrow \infty$ because of the divergence of the second derivative of the scale factor. Thus, a stronger singularity occurs in this class of models. Such divergence of the scalar curvature is not present in the simple quintessence case.

We have also shown the important role of the additional linear and quadratic terms of $t_{s}-t$ in the form of the scale factor as $t \rightarrow t_{s}$. However, in the scalar-tensor case the quadratic term becomes subdominant close to the singularity. 
For quintessence models, we derived relations of the Hubble parameter, $H^{2}(z)=\Omega_{0, m}(1+$ $z)^{3}\left[1-(1+z)^{3}\left(1+z_{0}\right)^{-3}\right]+(1+z)^{6}\left(1+z_{0}\right)^{-6} H_{0}^{2}$ (for the fluid case) and $H(z)=\frac{H_{0}(1+z)^{3}}{\left(1+z_{0}\right)^{3}}$ (for the no fluid case), close to the singularity. These relations may be used as observational signatures of such singularities in this class of models.

Interesting extensions of the present analysis include the study of the strength of these singularities in other modified gravity models e.g. string-inspired gravity, Gauss-Bonnet gravity etc. and the search for signatures of such singularities in cosmological luminosity distance and angular diameter distance data.

\section{Acknowledgments}

I would like to thank my collaborators S. Lola and L. Perivolaropoulos for their stimulating and fruitful contribution and collaboration that led to this work. I also thank the organizers for the opportunity to present these results and for the stimulating atmosphere they have created during the conference. Financial support from the COST Action CA15108, is gratefully acknowledged.

\section{References}

[1] E. J. Copeland, M. Sami, and S. Tsujikawa, "Dynamics of dark energy", Int. J. Mod. Phys. D 15, 1753 (2006).

[2] J. Frieman, M. Turner, and D. Huterer, "Dark energy and the accelerating universe", Annu. Rev. Astron. Astrophys. 46, 385 (2008).

[3] A. G. Riess et al. (Supernova Search Team), "Observational evidence from supernovae for an accelerating universe and a cosmological constant", Astron. J. 116, 1009 (1998).

[4] A. H. Jaffe et al. (Boomerang), "Cosmology from MAXIMA-1, BOOMERANG and COBE / DMR CMB Observations", Phys. Rev. Lett. 86, 3475 (2001).

[5] S. W. Hawking and G. F. R. Ellis, "The Large Scale Structure of Space-Time", Cambridge Monographs on Mathematical Physics, (Cambridge University Press, Cambridge, 2011).

[6] M. Davis, G. Efstathiou, C. S. Frenk, and S. D. M. White, "The evolution of large scale structure in a universe dominated by cold dark matter", Astrophys. J. 292, 371 (1985).

[7] S. Nojiri and S. D. Odintsov, "Introduction to modified gravity and gravitational alternative for dark energy, Theoretical physics: Current mathematical topics in gravitation and cosmology", Proceedings, 42nd Karpacz Winter School, Ladek, Poland, February 6-11, 2006, eConf C0602061, 06 (2006); Int. J. Geom. Methods Mod. Phys. 04, 115 (2007).

[8] S. Nojiri and S. D. Odintsov, "Modified gravity with negative and positive powers of the curvature: Unification of the inflation and of the cosmic acceleration", Phys. Rev. D 68, 123512 (2003).

[9] I. Zlatev, L.-M. Wang, and P. J. Steinhardt, “Quintessence, cosmic coincidence, and the cosmological constant", Phys. Rev. Lett. 82, 896 (1999).

[10] S. M. Carroll, "Quintessence and the rest of the world", Phys. Rev. Lett. 81, 3067 (1998).

[11] M. C. Bento, O. Bertolami, and A. A. Sen, "Generalized chaplygin gas, accelerated expansion and dark energy matter unification", Phys. Rev. D 66, 043507 (2002). 
[12] N. Bilic, G. B. Tupper, and R. D. Viollier, “Unification of dark matter and dark energy: The Inhomogeneous chaplygin gas", Phys. Lett. B 535, 17 (2002).

[13] R. J. Scherrer, "Phantom dark energy, cosmic doomsday, and the coincidence problem", Phys. Rev. D 71,063519 (2005).

[14] S. Nesseris and L. Perivolaropoulos, "The Fate of bound systems in phantom and quintessence cosmologies", Phys. Rev. D 70, 123529 (2004).

[15] L. Perivolaropoulos, "Constraints on linear negative potentials in quintessence and phantom models from recent supernova data", Phys. Rev. D 71, 063503 (2005).

[16] A. Lykkas and L. Perivolaropoulos, "Scalar-Tensor Quintessence with a linear potential: Avoiding the Big Crunch cosmic doomsday", Phys. Rev. D 93, 043513 (2016).

[17] "Mathematical Structures of the Universe", edited by S. J. Szybka, M. Eckstein, and M. Heller (Copernicus Center Press, Kraków, 2014), pp. 99-117.

[18] M. P. Dabrowski, “Are singularities the limits of cosmology?", arXiv:1407.4851.

[19] I. Antoniadis, J. Rizos, and K. Tamvakis, "Singularity-free cosmological solutions of the superstring effective action", Nucl. Phys. B415, 497 (1994).

[20] G. N. Felder, A. V. Frolov, L. Kofman, and A. D. Linde, "Cosmology with negative potentials", Phys. Rev. D 66, 023507 (2002).

[21] J. D. Barrow, "Sudden future singularities", Classical Quantum Gravity 21, L79 (2004).

[22] L. Fernandez-Jambrina and R. Lazkoz, "Geodesic behaviour of sudden future singularities", Phys. Rev. D 70, 121503 (2004).

[23] L. Fernandez-Jambrina and R. Lazkoz, "Classification of cosmological milestones", Phys. Rev. D 74, 064030 (2006).

[24] L. Perivolaropoulos, "Fate of bound systems through sudden future singularities", Phys. Rev. D 94, 124018 (2016).

[25] F. J. Tipler, "Singularities in conformally flat spacetimes", Phys. Lett. A 64, 8 (1977).

[26] A. Krolak, "Towards the proof of the cosmic censorship hypothesis", Classical Quantum Gravity 3, 267 (1986).

[27] S. Nojiri, S. D. Odintsov, and S. Tsujikawa, "Properties of singularities in the (phantom) dark energy universe", Phys. Rev. D 71, 063004 (2005).

[28] J. D. Barrow and A. A. H. Graham, "New singularities in unexpected places", Int. J. Mod. Phys. D 24, $1544012(2015)$.

[29] A. Lymperis, L. Perivolaropoulos, S. Lola "Sudden Future Singularities in Quintessence and Scalar-Tensor Quintessence Models", Phys.Rev. D96 (2017) no.8, 084024.

[30] J. D. Barrow and A. A. H. Graham, "Singular Inflation", Phys. Rev. D91, 083513 (2015).

[31] J. D. Barrow, "More general sudden singularities", Classical Quantum Gravity 21, 5619 (2004).

[32] J. D. Barrow and A. C. Ottewill, "The stability of general relativistic cosmological theory", J. Phys. A 16, 2757 (1983).

[33] F. Briscese, E. Elizalde, S. Nojiri, S.D. Odintsov, "Phantom scalar dark energy as modified gravity: Understanding the origin of the Big Rip singularity", Phys. Lett. B646, 105-111 (2007) 
[34] Shin'ichi Nojiri, Sergei D. Odintsov, "The Future evolution and finite-time singularities in $F(R)$-gravity unifying the inflation and cosmic acceleration", Phys. Rev. D78, 046006 (2008).

[35] Kazuharu Bamba, Shin'ichi Nojiri, Sergei D. Odintsov, "The Universe future in modified gravity theories: Approaching the finite-time future singularity", JCAP 0810, 045 (2008).

[36] Shin'ichi Nojiri, Sergei D. Odintsov, “The Oscillating dark energy: Future singularity and coincidence problem", Phys.Lett. B637, 139-148 (2006).

[37] Shin'ichi Nojiri, Sergei D. Odintsov, "Quantum escape of sudden future singularity", Phys.Lett. B595, 1-8 (2004).

[38] Alexander Yu. Kamenshchik, Ugo Moschella, Vincent Pasquier, "An Alternative to quintessence", Phys. Lett. B511, 265-268 (2001).

[39] Vittorio Gorini, Alexander Yu. Kamenshchik, Ugo Moschella, Vincent Pasquier, "Tachyons, scalar fields and cosmology", Phys.Rev. D69, 123512 (2004). 